Review

\title{
Research progress on the relationship between lung cancer drug-resistance and microRNAs
}

\author{
Xuan Ma, Ai-Ling Liang, Yong-Jun Liu ${ }^{凶}$ \\ Medical Molecular Diagnostics Key Laboratory of Guangdong \& Departments of Biochemistry and Molecular Biology \& Departments of Clinical Biochemistry, \\ Guangdong Medical University, 523808, Dongguan, Guangdong, P.R. China \\ $\square$ Corresponding author: liuyongjun@gdmu.edu.cn
}

(1) The author(s). This is an open access article distributed under the terms of the Creative Commons Attribution License (https://creativecommons.org/licenses/by/4.0/). See http://ivyspring.com/terms for full terms and conditions.

Received: 2018.12.02; Accepted: 2019.09.13; Published: 2019.11.17

\begin{abstract}
Lung cancer, a malignant tumor with the highest death rate of cancer, seriously endangers human health. And its pathogenesis and mechanism of drug resistance has been partially clarified, especially for the signal pathway of epidermal growth factor receptor (EGFR). The targeting therapy of EGFR signaling pathway in non-small cell lung cancer (NSCLC) has achieved a certain effect, but the two mutation of EGFR and other mechanisms of lung cancer resistance still greatly reduce the therapeutic effect of chemotherapy on it. MicroRNA is an endogenous non coding RNA, which has a regulatory function after transcriptional level. Recent studies on the mechanism of lung cancer resistance have found that a variety of microRNAs are related to the mechanism of lung cancer drug-resistance. They can regulate lung cancer resistance by participating in signal pathways, drug resistance genes and cell apoptosis, thus affecting the sensitivity of cancer cells to drugs. Therefore, microRNAs can be used as a specific target for the treatment of lung cancer and plays a vital role in the early diagnosis, prognosis and treatment of lung cancer. This article reviews the mechanisms of lung cancer resistance and its relationship with microRNAs.
\end{abstract}

Key words: microRNAs, lung cancer, drug-resistance, EGFR, p53, EMT, apoptosis

\section{Introduction}

In recent decades, lung cancer has become the most common malignant disease with the highest incidence and mortality rate in China and even the whole world, which poses a serious threat to human health. In 2016 Lachgar A et al [1] found that over $60 \%$ of lung cancer patients present with locally advanced or metastatic disease (stage III or IV) at the time of diagnosis, during this period that surgical resection may not be an option. Therefore, until recently, conventional chemotherapy and radiation therapy were the main types of treatments for lung cancer patients ${ }^{[2]}$. However drug resistance in chemotherapy for lung cancer is complex and these mechanisms are not clarified yet, which seriously affects patients' clinical treatment effect and cause a very poor prognosis.

Over the past few decades, non-small cell lung cancer (NSCLC) and small cell lung cancer (SCLC) were the most common classification of diagnosing lung cancer.

This way doesn't require further morphological sub classification because of the limited treatment options. NSCLC comprises approximately $80 \%-85 \%$ of all lung cancers including adenocarcinoma and squamous cell carcinoma [2,3]. Thus NSCLC becomes the focus of studying lung cancer resistance mechanisms.

Nowadays there are several mechanisms of lung cancer drug-resistance mentioned in various literatures. In 2015 Wang et al found EGFR self-resistance mutation, T790M second mutation and the activation of the PI3K/AKT signal pathways ${ }^{[4]}$. In 2007 Lim et al found that gene of phosphate and tension homology deleted on chromosome ten (PTEN) mutations and Axl overexpression [5]. In 2005 Pao et al found that kirsten rat sarcoma viral oncogene 
(KRAS), v-raf murine asrcoma viral oncogene homolog B1 (BRAF) and human epidermal growth factor receptor-2 (HER-2) mutations [6]. In 2007 Engelman found that mesenchymal-epithelial transition (MET) factor proto oncogene amplification and overexpression of protein ${ }^{[7]}$. There are also some mechanisms concerned with signal pathways. For example, in $2014 \mathrm{Wu}$ found that epithelialmesenchymal transition (EMT) cell growth related to the deletion of signal pathways ${ }^{[8]}$.

Most of the above-mentioned mechanisms of lung cancer resistance are related to gene mutation, deletion and amplification.

In addition to the above-mentioned lung cancer resistance mechanisms, there are other lung cancer resistance mechanisms involving pharmacokinetics and multidrug resistance genes, such as the following aspects. i) In 2016 Wei et al found that membrane transporter-mediated drug efflux pump mechanism, as the most common mechanism, which can reduce intracellular drug accumulation. This mechanism involves overexpressed membrane protein with efflux pump, such as ABC family member P-glycoprotein (P-gp), multidrug resistance-associated protein (MRP), lung resistance-related protein (LRP) and so on [9]. ii) Intracellular abnormal enzyme system can be combined with a variety of chemotherapeutic drugs, which reduces drug activity, or enhances the DNA repair ability of tumor cells, and prevent the damage of chemotherapeutic drugs to the tumor cells [10]. These intracellular abnormal enzymes are overexpressed topoisomerase, glutamyl transpeptidase, $\mathrm{O}^{6}$-methyguanine-DNA methyltransferase (MGMT) and so on. iii) In 2015 Javid et al found that anti-apoptotic effect of cells is enhanced, such as over-expression of anti-apoptotic genes bcl-2 and c-myc, which makes tumor cells less susceptible to apoptosis [11].

The epidermal growth factor receptor (EGFR) is a member of a family of four closely related receptors: EGFR (or erbB1), HER2/neu (erbB2), HER3 (erbB3) and HER4 (erbB4) ${ }^{[12]}$. Approximately $50 \%$ of Asian patients with NSCLC have EGFR mutations [13]. Patients with sensitizing mutations of EGFR, compared to conventional chemotherapy, these targeted therapy, such as epidermal growth factor receptor-tyrosine kinase inhibitors (EGFR-TKIs) have achieved huge success and became one of the standard treatment-regimes for NSCLC. However, patients ultimately develop resistance to these drugs, even though EGFR-TKIs have been established as the standard therapy for EGFR-sensitizing mutant advanced NSCLC clinically $[14,15]$.

According to the current mechanisms of lung cancer resistance, researchers have classified them into two categories: primary and acquired resistance. The primary resistance is defined as the failure to respond to the treatments at the first time after receiving EGFR-TKIs and presents no obvious improvement in symptoms, disease control or overall survival. Approximately $85 \%$ of all primary lung cancers are NSCLC. While some patients with activating EGFR mutations who are initially responsive to EGFR TKIs very well at firstly, eventually would be developed acquired resistance after a complete or partial response or $\geq 6$ months of stable disease after treatment with a targeted therapy [16]. Strategies to overcome these intrinsic and acquired resistance mechanisms are complex. Thus studies on the lung cancer drug resistance are extremely important in order to define the best treatment strategy.

MicroRNAs (miRNAs, miRs) are a class of small endogenous single strand non-coded RNA, about 19-25 nt, found in eukaryotes, and have the function of regulating after gene transcription [15]. MicroRNAs' regulation mechanism is that the longer miRNAs primary transcript produces mature miRNAs through a series of nuclease shear processing. Then these mature miRNAs are assembled into the RNA-induced silencing complex (RISC). Then mature miRNAs recognize and combine the target genes or the target mRNAs' 3 ' terminal non translation region (UTR) by complementary pairing of bases. The RISC will conduct the process of mRNAs' degradation or inhibition of their translations. MiRNAs and target mRNAs combined incompletely can inhibit the expression of mRNAs at the level of protein translation. While when they may complement each other completely (or almost completely complementary), which can degrade the target mRNAs degradation and change the expression level of the target protein, then achieve the regulatory effect [17].

In 2016 Ren et al found that miRNAs can regulate a variety of target genes or one kind of miRNAs can be regulated by various genes [18]. In particular, in 2006 George et al found that abnormal expression of miRNAs is probably one of the causes of tyrosine kinase inhibitors (TKIs) underlying mechanisms of primary resistance [19]. In 2016 Thomas et al also found that several miRNAs can increase the therapeutic efficiency of EFGR-TKIs by regulating the drug sensitivity of cancer cells [20].

From the above literatures, it can be seen that miRNAs may play an important role in the treatment of lung cancer by regulating genes' expression, or by affecting the efficacy of drugs. Therefore, this article mainly reviews the role of miRNAs in several lung cancer resistance mechanisms based on recent literatures. 


\section{Mutual regulation between overexpression of AXL and miRNAs in NSCLC}

Axl (also known as AXL, UFO, ARK, or TYRO7) is one of the members of the receptor tyrosine kinase sub-family, and binds to the growth stagnation specific gene 6 (Gas6) to activate its tyrosine kinase activity. It mediates signal conduction from extracellular matrix to cells, and activates downstream signal transduction pathways, and participates in cell adhesion, proliferation, and apoptosis. The abnormal expression of Axl gene plays an important role in the tumorigenesis and development of many tumors [21].

In 2016 Chen found that miR-432 over-expression can increase the sensitivity of lung adenocarcinoma cells to cisplatin. E2F3 and AXL are targets for miR-432 acting on lung adenocarcinoma, and the target is $3^{\prime}$ UTRs. It was found that miR-432 levels were negatively correlated with the levels of E2F3 and AXL. Increase miR-432 may reverse the resistance of lung adenocarcinoma cells to cisplatin by targeting to improve the therapeutic efficacy of lung adenocarcinoma patients [22].

In addition, in 2014 Wang et al found that overexpression of Axl significantly increased the expression level of miR-374a and decreased the expression of miR-548b in gefitinib-resistant NSCLC cells, Calu1 and HCC827-Gef cells. Then they found miR-374a induces EMT, colony formation and drug resistance in gefitinib-sensitive lung cancer cells. While silencing of miR-374a inhibits migration and invasion in gefitinib-resistant NSCLC. And miR-548b can target CCNB1 to control cell cycle progression in NSCLC cells [23]. Following that we can infer that overexpression of Axl could increase drug resistance to gefitinib in NSCLC cells via mediating miRNAs involved with EMT or cell proliferation process.

In 2016 Cho et al had another interesting founding. They demonstrated that there may be a regulatory feedback loop between AXL and miR-34a at the post-transcriptional level. Both the GAS6-binding domain and the kinase domain of AXL are shown to be crucial for this autoregulation. They used bioinformatics and molecular techniques to reveal that miR-34a may target the 3' UTR of AXL mRNA to inhibit AXL express. Importantly, AXL overexpression may induce miR-34a expression by activating the transcription factor ELK1 via the JNK signaling pathway. That subsequently represses AXL expression to induce G1 arrest and cell apoptosis. Therefore, they assume not only that AXL could up-regulate miR-34a expression, but also that miR-34a could turn back to down-regulate AXL by directly targeting its 3' UTR [24].
It has been reported that AXL transcription can be induced by MAPK-AP1 activation and by MZF1 transcriptional activity in EGFR-TKIs [25]. Axl may drive cancer cell growth through activation of several downstream pathways, such as MAPK, AKT and NF-kB, and Axl-mediated EGFR-TKI acquired resistance may occur during EMT [23]. In 2012 Zhang et al also reported that AXL upregulation is the second most common mechanism of EGFR TKI acquired resistance (after EGFR T790M) in EGFR-mutant NSCLCs [26].

Based on the above literature, we can found that over-expressed Axl can not only regulate miRNAs or be regulated by miRNAs in an ex parte way, but also contrast a feedback loop with miRNAs to regulate each other's expression. This finding is interesting and significant because it offers another possibility in treating lung cancer patients. If the feedback loop really exists between miRNAs and targeted genes, we'd better avoid using this kind of miRNAs or targeted gene medicines singly, which may reduce the effect of the treatment in lung cancer. And this feedback loop explains the drug resistance mechanisms from another point of view, making it more completely. With the deepening of research on lung cancer, these findings provide a new way to understand the mechanisms of drug resistance in lung cancer cells.

\section{MiRNAs can mediate the deletion of PTEN to lead cell resistance}

PTEN (or named mutated in multiple advanced cancers, MMAC1) is a tumor suppressor gene. It is the key regulator of cell proliferation, growth, differentiation and apoptosis [27]. As early as $1994 \mathrm{Xu}$ et al have found that the loss of PTEN is associated with the development and process of cancer [28]. In 2006 Tang et al found that PTEN expression is down or absent in NSCLC [29].

PTEN enzymes are involved in transduction of signaling pathways. PTEN can transmit signals to cells, stop cell division and enter programmed cell death (or apoptosis). PTEN can catalyze PIP3 dephosphorylation. When PTEN is absent, PIP3 dephosphorylation is weakened, further activating PI3K/AKT pathway and inducing drug resistance. In 2010 Yamamoto found that the deletion of PTEN is due to the EGR1 shift of transcription factor PTEN, which is controlled by nuclear and further causes TKI resistant [30]. In 2011 Derfoul et al found that miR-214 can regulate the tumor suppressor gene PTEN and regulate the proliferation and apoptosis of tumor cells. Inhibition of PTEN activates the PI3K/AKT pathway, thereby increasing the survival of tumor cells treated with drug chemotherapy [31]. 
MiR-92a has been proved to play an important role in many kinds of human cancers. In 2016 Ren et al found that miR-92a can promote cell proliferation, migration and invasion of NSCLC, and increase the drug resistance of NSCLC to chemotherapeutic drugs. Meanwhile the downregulation of miR-92a will have the opposite effect.

The researchers found that the miR-92a expression was higher in four different NSCLC cell lines (A549, SPCA1, H1299 and H358) than that in normal lung cells. By constructing the reported gene plasmid and transfecting it into the A549 cells, the researchers conducted Luciferase assay. They found that the expression of PTEN and miR-92a was negatively correlated. At the same time, Quantitative realtime reverse transcription PCR and Western blotting experiments also found that the levels of PTEN mRNA and protein in A549 cells were negatively correlated with the expression of miR-92a. And the relevant levels of PI3k and AKT were also negatively related to miR-92a. Therefore, it is inferred that PTEN is a direct target gene for miR-92a. The expression of miR-92a is increased when PTEN is deleted, meanwhile the PTEN of phosphorylation is reduced, and the PI3K/Akt signal pathway is activated to lead to drug resistance of lung cancer cells ${ }^{[18]}$.

In 2015 Xie et al transfected miR-106a mimics and knocked down miR-106a in three kinds of NSCLC cells to apply experiments. They found that miR-106a was up-regulated in NSCLC cell lines. Inhibition of miR-106a in NSCLC cells substantially inhibited cell proliferation, migration, and invasion. PTEN as the direct target of miR-106a, it was inversely correlated with PTEN in NSCLC cells. Their study demonstrated that miR-106a was significantly increased in NSCLC cell lines. Down-regulated expression of miR-106a could inhibit tumor growth and metastasis of NSCLC cells by increasing PTEN expression [32]. A previous report showed that the PTEN/PI3K/pAkt pathway may play an important role in lung cancer [33].

Taken together, these findings indicated that miRNAs could promote growth, metastasis, and chemoresistance in NSCLC cells at least partially by targeting PTEN. Until now, drug resistance mechanisms in lung cells referred to the deletion of PTEN, at least PI3K/Akt signaling pathway is of great significance in this part. While as for the drug resistance in lung cancer, there must be more profound and complicated causes involved in the mechanisms need to be explored in the future.

\section{MiRNAs mediate cell resistance in lung cancer via apoptosis}

Apoptosis is a gene-encoded programmed cell death. Under various physiological conditions, it is regulated by intracellular and/or extracellular signals and characterized by morphological changes of the cells targeted for death [34].

Caspase family includes apoptotic initiators in the upstream of the cascade reaction, such as Caspase-2, Caspase-8, Caspase-9, and Caspase-10 and so on. These factors can self-activate and then identify and activate downstream caspase members with the help of other proteins. For example, Caspase- 8 can activate Caspase members in the downstream of almost all apoptosis cascade reactions and induce apoptosis, which lead to cascade amplification effect of apoptosis [35, 36].

$\mathrm{Bcl}-2$ and NF- $\mathrm{kB}$ are recognized as anti-apoptosis genes. The Bcl-2 family contains at least 17 or more members. A large number of studies have confirmed that there are Bcl-2 and NF- $\mathrm{kB}$ super-expressions in lung cancer, gastric cancer, ovarian cancer, and colon cancer. Bcl-2 protein is a product of Bcl-2 pro-oncogene encoding, which promotes the survival of cancer cells and inhibits apoptosis. Their anti-apoptosis effects play an important role in the malignant proliferation of tumor cells and are closely related to invasion and metastasis $[37,38]$.

According to the structure and function, the Bcl-2 family can be divided into three groups. The first group is anti-apoptosis or apoptosis family, including Bcl-2, Bcl-XL, Mcl-1, Bcl-w and A1. They all contain 4 $\mathrm{BH}$ domains (BH1-4). The second group is multiple $\mathrm{BH}$ domain apoptosis family, including Bax and Bak, which contains multiple $\mathrm{BH}$ domains. The third group is only $\mathrm{BH} 3$ domains families which promote apoptosis. They contain only $\mathrm{BH} 3$ domains that are homologous to Bcl-2, including Bad, Bid, Bik, Bim, Bmf, Hrk, Noxa, PUMA, and Beclin-1 [39]. Bax and Bak's BH3 domains can form heterodimers with Bcl-2 and Bcl-xl, promoting apoptosis [40]. At the protein level, different Bcl-2 family proteins can interact to form different dimers, thus antagonizing or enhancing the function of apoptosis [41]. Therefore, some researchers proposed that the ratio between inhibition factors and activation factors may determine the tendency of cells to apoptosis [42, 43]. When apoptosis is suppressed, it may lead to malignant lesions and drug resistance in cancers. Under poor conditions (such as DNA damage or hypoxic oxygen), tumor suppressor genes such as P53 can trigger apoptosis ${ }^{[44]}$.

P53 is one of the most common mutation genes in human tumors. P53 gene mutations and functional defects are about $45 \%-70 \%$ in NSCLC. It is an important cause of tumor occurrence and treatment failure [45]. Wild p53 function may be regulated by p53 apoptosis stimulating protein. The latter protein combines with P53 to form a complex to act on the 
original apoptosis gene. Then that promotes p53 dependent apoptosis by increasing the activity of the original apoptosis gene [46].

The process of apoptosis is complex and involves a variety of protein families, aforementioned caspase family proteins, the Bcl-2 family proteins, and the p53 protein, which can be divided into two categories according to their functions in apoptosis anti-apoptosis and pro-apoptosis. We can find that these proteins could be mediated by miRNAs to perform the two different functions to regulate the process of drug resistance in lung cancer.

Caspase- 3 is one of the most important executors of the caspase family. It is the main effect factor in the apoptosis process. Its activation is a sign that apoptosis has entered an irreversible stage ${ }^{[47]}$. There is a study found that the activity of cysteine caspase- 3 in NSCLC cells after transfection of miR-92a mimics was significantly reduced. And the activity of caspate- 3 was significantly enhanced after transfection of anti-miR-92a. It is shown that miR-92a can promote cell proliferation and reduce apoptosis in NSCLC. It is speculated that overexpressed miR-92a can increased the resistance of NSCLC cells through down-regulated caspase family related genes [18].

In 2015 Cui et al demonstrated that miR-244 markedly enhanced proliferative and migratory effect of NSCLC cells. They found the expression of miR-224 negatively correlates with the expression of Caspase-7 and Caspase- 3 in tissue samples from patients with lung cancer, which also induced drug resistance in NSCLC. However miR-224 regulates Caspase-3 at translation levels and Caspase-7 in lung cancer remains largely unexplored. At least miR-224 promotes lung cancer cell growth and migration partially by inhibiting Caspase-7. Then they also confirmed that up-regulated miR-224 expression in NSCLC might be partially controlled by NF-kB signaling through binding of RELA/p65 to miR-224 promoter region. Hence, they suggest that NF-kB/miR-224/CASP-3, -7 pathway might be an ideal target for therapeutic intervention in certain lung cancer patients ${ }^{[48]}$.

In $2016 \mathrm{Wu}$ et al found that miR-301b was highly expressed in lung cancer tissues and cell lines. Expression of miR-301b was induced by hypoxia, and miR-301b suppressed expression of Bim by targeting its $3^{\prime}$ UTR. Their results demonstrated that hypoxia-induced microRNA-301b expression enhances cell growth and reduces apoptosis by regulating Bim expression [49].

Erk1/2 pathway controls several cellular functions, such as proliferation, survival and migration, these all are closely related to drug resistance in cancer [50]. In 2012 Romano et al showed a link between the Erk1/2 pathway and Bim expression through miR-494 in NSCLC. MiR-494 was the most down-regulated miRNA after Erk1/2 inactivation. They assumed that axis PED-ERK-miR-494-BIM existed in NSCLC cells. And then they found that the overexpression of this microRNA in H460 tumor necrosis factor (TNF)-related apoptosis-inducing ligand (TRAIL)-sensitive cells, by down-regulating BIM, increased the resistance to TRAIL induced apoptosis. The same result was obtained on TRAIL-resistant A549 cells, the down-regulation of miR-494 made A549 more sensitive to TRAIL-induced apoptosis, confirming the relevant role of miR-494 in TRAIL resistance. That means miR-494 could induce TRAIL resistance through the down-modulation of Bim to increase resistance in NSCLC. [51].

In 2018 Choi et al found expression of P53, and Notch2 were decreased in miR-93-5p over-expressed lung cells. And expression of Bcl-w or P21 was also negatively correlated with that of miR-93-5p in lung cancer tissue samples [52]. The tumor suppressor p53 binds the promoting survival Bcl-2 family proteins such as Bcl-w [53]. We can infer that there is a Bcl-w-P53/Notch2-P21 signaling axis to mediate cells growth and premature senescence in lung cells. These cell behaviors may furthermore increase drug resistance in lung cancer.

In 2018 Baumgartner et al explored 17 miRNAs are dysregulated following PI3K/AKT inhibition of EGFR mutant NSCLC cells. They used bioinformatics to analyze then they found that dysregulated miRNAs act in a concerted manner to enhance the activity of the EGFR signaling pathway, such as PI3K/AKT, KRAS/ERK and JAK/STAT signal pathways. These findings were closely mirrored by attenuation miR-19b in NSCLC cell lines which resulted in reduced phosphorylation of ERK, AKT and STAT and effector proteins in EGFR mutant NSCLC cells.

They found that serine/threonine phosphatase PP2A subunit PPP2R5E and BCL2L11 encoding Bim were identified as major targets of miR-19b by target validation assays. MiR-19b potentiates EGFR signaling by targeting PP2A subunit PPP2R5E and confers apoptosis resistance by targeting BCL2L11 encoding the $\mathrm{BH} 3$ domain containing protein Bim. MiR-19b induces proliferation and apoptosis resistance by targeting PPP2R5E and BCL2L11. It can be inferred that molecular processes of EGFR signaling involving miRNAs may provide insights into improving the management of EGFR-mutant lung cancer patients treated with TKIs [54]. The proapoptotic BH3-only protein Bim is a master regulator of cell death in cancer cells [55], which is a relevant target of miR-19b in spontaneous and decrease drug resistance in lung cancer. 
From the above literature, we can see that miRNAs can regulate the relevant apoptosis proteins or genes at different nodes in signaling pathways through their own over-expression or downward expression. These apoptosis family members, such as Bim, P53 and Caspase3 and the like, have already been a research hot topic for a long time, and some mechanisms are pretty clear now. The decrease of apoptotic ability is accompanied by the increase of drug resistance in lung cancer cells. The drug resistance of lung cancer cells is regulated by enhancing the ability of cells to anti-apoptosis or pro-apoptosis. The role of cell apoptosis in drug resistance of lung cancer has not been elucidated until now, however these documents are sufficient to indicate a relationship between both. As the research further develops, we can use apoptosis to regulate miRNAs to make lung cancer cells resensitive to drugs, improving efficacy of medicine in lung cancer patients.

\section{MiRNA-200 family mediate drug resistance by EMT in lung cancer}

EMT is a fundamental cellular process that plays critical roles in development, cancer metastasis and tissue wound healing [56]. In the process of drug resistance, the morphological changes of epithelial cells to mesophyll cells are often accompanied by the decrease of epithelial markers and the increase of mesophyll markers [57]. At the same time, it is often accompanied by Axl activation, transformation growth factor- $\beta$ (TGF- $\beta$ ) activation [58]. ZEB1 (zinc finger E-box binding homeobox 1 ) is the key molecule for EMT conversion, an important activator in EMT inhibiting expression of basement membrane components and cell polarity factors [59]. And EMT is characterized by a switch from an epithelial phenotype of polarized cells with expression of epithelial markers such as E-cadherin to a mesenchymal phenotype of cells that lack polarity, are motile and have down regulation of E-cadherin expression accompanied by an increase in Vimentin [60, 61].

As early as 1994, Nichterlein et al found that miR-452 can exert anti-tumor activity by inhibiting the EMT of NSCLC cells [62]. In 2011 Roybal et al found that miR-200 can inhibit the invasion and expression of lung adenocarcinoma cells by inhibiting the expression of target gene Flt1/VEGFR1 [63]. The EMT process and sensitivity to EGFR-TKIs therapy of bladder cancer cells can be regulated by regulating the expression of target gene ERRFI-1 [64]. In another study, researchers screened miRNAs associated with kzoltinil resistance H1322 cells in NSCLC found that the reduction of miR-200c was likely related to EMT [65]. About $20 \%-44 \%$ of treated NSCLC patients acquire resistance to EGFR-TKIs through phenotypic changes of the tumor cells undergoing EMT, possibly as a result of altered epigenetic regulation [66]. It has been shown that EMT-related acquired resistance to EGFR-TKIs in NSCLC is driven by increased ZEB1, which is negatively regulated by miR-200c [67, 68].

In a recent study, miR-200c and Cathepsin L were proved to reciprocally influence each other, regulating paclitaxel resistance through EMT in A549 cells [69]. In 2013 Ahmad A et al found that pretreatment with miR-200b and let-7c in TGF- $\beta$ 1-induced A549 cells, about $67.69 \%$ inhibition of TGF$\beta$ 1-mediated effect on erlotinib resistance was observed. Re-expressing of miR-200b and let-7c could also downregulate ZEB1 and remarkably break-off the inhibition of E-cadherin expression, both of which were the index to the reversal of EMT [60]. Except erlotinib, gefitinib is another EGFR-TKI that is often used in TKI therapies to treat NSCLC patients. And in 2015 Zhen $Q$ et al found that the expression of miR-200a was observed to render NSCLC cells much more sensitive to gefitinib treatment [70].

MiR-200 is a family of tumor suppressor miRNAs consisting of five members, which are significantly involved in inhibition of EMT, repression of cancer stem cells (CSCs) self-renewal and differentiation, modulation of cell division and apoptosis, and reversal of chemoresistance [71]. Phenotypic changing is one of the characters of cancer cells, such as EMT. It is because of the appearance of this kind of biological behavior, which makes cancer cells get the greater ability of migration and invasion. So the cancer cells are more likely to develop resistance. From the above literates we can found the countless ties between the EMT and the miR-200 family, it can be inferred that EMT plays an important role in lung cancer resistance, but the specific mechanism has not been clarified. Besides miR-200 family, it is also not known whether EMT is related to other genes or miRNAs. But it's more important to focus on research about miR-200 family; this may reveal the root cause of EMT, to solve the problem of cancer cell migration and invasion at the source. If it can be targeted to inhibit EMT through miRNAs, it may be possible to find new treatments for resistance to lung cancer, making the most of medicine treatment.

\section{MiRNAs and other resistance mechanisms in lung cancer}

\section{The mechanism of universal lung cancer resistance and miRNAs}

As mentioned earlier, the universal drugresistance mechanism for lung cancer involves drug metabolic kinetics, which includes MRP, LRP and 
Breast cancer resistant protein (BCRP). These four membrane transporters cause drug resistance by reducing intracellular drug concentration or changing the intracellular distribution of drugs [72].

$\mathrm{LRP} / \mathrm{MVP}$ is a ribonucleoprotein particle able to transport drugs such as platinum derivatives from the nucleus to the cytoplasm. After that, these compounds can be transported outside from the cells by ABC-transporters [73-75]. In 2016 Janikova et al immunohistochemically evaluated expression of P-gp, MRP and LRP/MVP and quantified the relative levels of miR-23b in 62 NSCLC patients' samples. Their results showed that miR-23b is mostly downregulated in NSCLC samples (57/62). Their findings indicate that the risk of death or relapse in NSCLC patients with downregulated miR-23b increases together with LRP/MVP expression and decreases in patients with upregulated miR-23b [76]. Expression of P-gp, MRP and LRP/MVP are often observed in NSCLC samples and it is believed that these molecules play a major role in the emergence of multidrug resistance ${ }^{[77-79]}$.

\section{APC methylation or involvement in lung cancer resistance}

The APC (adenomatous polyposis coli) gene can control the death or growth of cells. This gene encodes a tumor suppressor protein that acts as an antagonist of the Wnt signaling pathway. It is also involved in other processes including cell migration and adhesion, transcriptional activation, and apoptosis [80]. Abnormal methylation of APC gene promoter occurs in lung cancer patients. In 2016 Feng et al found that APC gene promoter methylation is associated with the clinical characteristics of tumor patients, mainly tumor staging, lymph node metastasis and smoking [81]. While in NSCLC $\beta$-catenin and APC mutations are uncommon [82].

Resistance of the same cell line to paclitaxel was significantly associated with miR-135a expression. In both in vitro and in vivo models, researchers observed that inhibition of miR-135a expression led to re-sensitization of previously resistant NSCLC cells to paclitaxel and caused the cells to undergo apoptosis. Expression of miR-135a has also been linked to the activity of the APC gene, which is involved in cancer development ${ }^{[83]}$.

\section{Resistance to lung cancer related to TRAIL}

TRAIL, a cytokine and a member of the TNF family, is being tested in clinical trials as a powerful anticancer agent. Although TRAIL had shown clinical efficacy in a subset of NSCLC patients, acquired resistance to this anticancer agent undermines its therapeutic value. The mechanism of this resistance is still not fully understood [84].

In 2008, Garofalo et al reported that NSCLC cells overexpressed miR-221/222 were TRAIL-resistant and showed an increase in migration and invasion capabilities [85]. Later on, the same group demonstrated that miR-34a and miR-34c, which are downregulated in NSCLC cell lines, could play a significant role in lung carcinogenesis by modulating the expression of PDGFR- $\alpha / \beta$ (platelet derived growth factor receptor- $\alpha / \beta$ ) and thereby regulating TRAIL-induced cell death sensitivity [86]. Expression of miR-21, miR-30c and miR-100 in NSCLC has been related to acquire TRAIL resistance [87]. Accordingly, continuous exposure to TRAIL caused acquired resistance to this agent in the future.

\section{Low expression of ATM could increase sensitivity to drugs in lung cancer}

The ataxia telangiectasia-mutated gene (ATM) is an important gene related to DNA damage testing. The ATM protein and the closely related kinase ATR (ataxia telangiectasia and Rad3 related, ATR) are thought to be master controllers of cell cycle checkpoint signaling pathways that are required for cell response to DNA damage and for genome stability. It directly perceives DNA double-strand fracture damage and initiates many DNA damage signal response pathways. P53 can be activated by phosphorylation and then transcribed to activate the expression of cell cycle checkpoint protein P21 to participate in the process of regulating DNA damage and repair [88]. In 2017 Allison Stewart et al found that schlafen family member 11 (SLFN11), EMT, and ATM mediate therapeutic response in SCLC to novel targeted agents such as PARP inhibitors. They found that knockdown of SLFN11 and ATM directly alters drug sensitivity. Silencing of ATM increased the sensitivity of DMS79 and H209 cells to cisplatin, talazoparib and olaparib in SCLC [89].

The drug resistance mechanism of lung cancer is complex and involves many proteins, genes, and miRNAs. A couple of examples above are all novel drug resistance mechanisms related to lung cancer. For instance, PDGFR- $\alpha / \beta$ is a kind of platelet-derived growth factor. This gene is essential for normal development of the cardiovascular system and aids in rearrangement of the actin cytoskeleton. Another one, APC gene is involved in Wnt signal pathway, which is related to the origin of cancer in humans. All of the above provide new perspectives for the study of drug resistance of lung cancer. It is believed that with the deepening and expansion of research, the roles of more and more genes, proteins and miRNAs can be elucidated in the future, to provide effective evidence for future drug resistance of lung cancer. 


\section{Summary}

In the lung cancer, miRNAs perform dual regulatory function: they act as oncogenes to promote cancer development or as tumor suppressors to inhibit this process [84]. As stated above miRNAs overexpressed in lung cells (or drug-resistance lung cells), such as miR-374a, miR-92a, miR-106a, act as oncogenes that promote the development of lung cancer cells and increase drug resistance by negatively regulating tumor suppressor genes and/or genes that control cellular processes such as EMT and apoptosis. Other miRNAs do the opposite function when they are overexpressed in the lung cells, such as miR- $548 \mathrm{~b}$, miR-200a, and miR-432. And there are some miRNAs such as miR-34a can form a negative feedback closedloop with the expression of the related gene Axl. And miR-34a and Axl are reciprocally linked in a feedback loop to regulate each other in the lung cancer cells, which can affect drug resistance of lung cancer cells.
The various miRNAs expression patterns are unique for specific resistance mechanisms in lung cancer. These molecules are either over- or under expressed depending on the resistance mechanism types ${ }^{[0,}{ }^{91]}$. Table 1 summarizes the effects of miRNAs in resistance mechanisms of lung cancer in this review.

The relationship and mechanism of miRNAs with lung cancer resistance is complex. And many drug resistance mechanisms have not been elucidated. A lot of experimental evidence suggests that single mechanism is not fully enough to explain the emergence of lung cancer resistance. According to the above literature, we can find that these lung cancer drug resistances are often concerned with many other mechanisms, such as pharmacokinetics, EMT, cell cycle and cell apoptosis and so on (Table 2). These factors interact with each other, leading to drug resistance in lung cancer.

Table 1. Effects of miRNAs in resistance mechanisms of lung cancer

\begin{tabular}{|c|c|c|c|c|c|c|c|}
\hline miRNA & $\begin{array}{l}\text { miRNA } \\
\text { expression } \\
\text { quantity }\end{array}$ & $\begin{array}{l}\text { Downstream } \\
\text { factors(target } \\
\text { genes) }\end{array}$ & $\begin{array}{l}\text { Regulatory } \\
\text { relationship with } \\
\text { downstream factors }\end{array}$ & Mechanism & Response to drugs & cell & References \\
\hline miR-432 & over-expression & E2F3 and AXL & inverse correlation & $\begin{array}{l}\text { arresting cell cycle } \\
\text { into } S \text { phase }\end{array}$ & $\begin{array}{l}\text { increased sensitivity to } \\
\text { cisplatin }\end{array}$ & $\begin{array}{l}\text { lung adenocarcinoma } \\
\text { cells }\end{array}$ & [22] \\
\hline miR-374a & $\begin{array}{l}\text { over-expression } \\
\text { because of AXL }\end{array}$ & $\begin{array}{l}\text { AXL (over- } \\
\text { expressed) }\end{array}$ & positive correlation & $\begin{array}{l}\text { induce EMT, } \\
\text { migration, invation, } \\
\text { colony formation }\end{array}$ & $\begin{array}{l}\text { increased resistance to } \\
\text { gefitinib }\end{array}$ & $\begin{array}{l}\text { gefitinib-resistant } \\
\text { NSCLC cells, Calu1 and } \\
\text { HCC827-Gef cells }\end{array}$ & [23] \\
\hline $\operatorname{miR}-548 b$ & $\begin{array}{l}\text { down } \\
\text {-expression } \\
\text { because of } \mathrm{AXL}\end{array}$ & $\begin{array}{l}\text { AXL (over- } \\
\text { expressed) }\end{array}$ & inverse correlation & $\begin{array}{l}\text { induce EMT, } \\
\text { migration, invation, } \\
\text { colony formation }\end{array}$ & $\begin{array}{l}\text { increased resistance to } \\
\text { gefitinib }\end{array}$ & $\begin{array}{l}\text { gefitinib-resistant } \\
\text { NSCLC cells, Calu1 and } \\
\text { HCC } 827-\text { Gef cells }\end{array}$ & [23] \\
\hline miR-548b & over-expression & CCNB1 & inverse correlation & cell cycle & $\begin{array}{l}\text { increased sensitivity to } \\
\text { gefitinib }\end{array}$ & NSCLC cells & [23] \\
\hline miR-34a & $\begin{array}{l}\text { over-expression } \\
\text { because of AXL }\end{array}$ & AXL & $\begin{array}{l}\text { mutual negative } \\
\text { adjustment }\end{array}$ & $\begin{array}{l}\text { JNK/ELK1 signaling } \\
\text { pathway, cell cycle } \\
\text { and cell apoptosis }\end{array}$ & $\begin{array}{l}\text { increased sensitivity to } \\
\text { drugs }\end{array}$ & $\begin{array}{l}\text { lung adenocarcinoma } \\
\text { cells (CLI cell lines) }\end{array}$ & [24] \\
\hline miR-92a & over-expression & PTEN & inverse correlation & $\begin{array}{l}\text { PI3K/Akt signal } \\
\text { pathway }\end{array}$ & increased resistance to drugs & $\begin{array}{l}\text { four different NSCLC } \\
\text { cell lines (A549, SPCA1, } \\
\text { H1299 and H358) }\end{array}$ & {$[18]$} \\
\hline miR-92a & over-expression & caspase-3 & inverse correlation & cell apoptosis & increased resistance to drugs & NSCLC cells & [18] \\
\hline miR-106a & over-expression & PTEN & inverse correlation & $\begin{array}{l}\text { PI3K/Akt signal } \\
\text { pathway }\end{array}$ & increased resistance to drugs & $\begin{array}{l}\text { three kinds of NSCLC } \\
\text { cells }\end{array}$ & [32] \\
\hline miR-244 & over-expression & $\begin{array}{l}\text { Caspase- } 7 \text { and } \\
\text { caspase- } 3\end{array}$ & inverse correlation & $\begin{array}{l}\text { NF-kB signal } \\
\text { pathway }\end{array}$ & increased resistance to drugs & NSCLC cells & [48] \\
\hline miR-494 & over-expression & Bim & inverse correlation & $\begin{array}{l}\text { MEK/ERK or Erk1/2 } \\
\text { pathway(PED-ERK- } \\
\text { miR-494-BIM) }\end{array}$ & $\begin{array}{l}\text { increased resistance to } \\
\text { TRAIL }\end{array}$ & NSCLC cells & [51] \\
\hline miR-93-5p & over-expression & $\begin{array}{l}\text { Bcl-w, P21, } \\
\text { P53 }\end{array}$ & inverse correlation & cell apoptosis & increased resistance to drugs & $\begin{array}{l}\text { lung cancer tissue } \\
\text { samples }\end{array}$ & {$[52,53]$} \\
\hline miR-19b & over-expression & $\begin{array}{l}\text { PPP2R5E and } \\
\text { BCL2L11 }\end{array}$ & inverse correlation & $\begin{array}{l}\text { EGFR mutation and } \\
\text { cell apoptosis }\end{array}$ & increased resistance to TKIs & NSCLC cells & [55] \\
\hline miR-200c & Low-expresstion & ZEB1 & inverse correlation & EMT & $\begin{array}{l}\text { increased resistance to } \\
\text { EGFR-TKIs }\end{array}$ & NSCLC cells & {$[64,66,67]$} \\
\hline miR-200c & over-expression & $\begin{array}{l}\text { Cathepsin } \\
\text { L(CTSL) }\end{array}$ & $\begin{array}{l}\text { mutual negative } \\
\text { adjustment }\end{array}$ & EMT & $\begin{array}{l}\text { increased sensitivity } \\
\text { to paclitaxel }\end{array}$ & A549 cells & {$[68]$} \\
\hline $\begin{array}{l}\text { miR-200b } \\
\text { and let-7c }\end{array}$ & over-expression & $\begin{array}{l}\text { ZEB1and } \\
\text { E-cadherin }\end{array}$ & inverse correlation & EMT & $\begin{array}{l}\text { increased resistance to } \\
\text { erlotinib }\end{array}$ & NSCLC cells & [69] \\
\hline miR-23b & low-expresstion & LRP/MVP & inverse correlation & $\begin{array}{l}\text { drug metabolic } \\
\text { kinetics }\end{array}$ & increased resistance to drugs & NSCLC samples & {$[76-79]$} \\
\hline miR-135a & low lexpresstion & APC gene & inverse correlation & cell apoptosis & $\begin{array}{l}\text { increased sensitivity } \\
\text { to paclitaxel }\end{array}$ & NSCLC cells & [83] \\
\hline $\begin{array}{l}\operatorname{miR}-34 a \\
\text { and } \\
\text { miR-34c }\end{array}$ & over-expression & PDGFR- $\alpha / \beta$ & inverse correlation & cell apoptosis & $\begin{array}{l}\text { increased sensitivity } \\
\text { to TRAIL }\end{array}$ & NSCLC cell lines & [86] \\
\hline
\end{tabular}


Table 2. Drug resistance mechanisms in lung cancer

\begin{tabular}{|c|c|c|}
\hline Drug resistance mechanisms & Cause of drug resistance & References \\
\hline EGFR self-resistance mutation & Gene mutation & [4] \\
\hline T790M second mutation & Gene mutation & [4] \\
\hline $\begin{array}{l}\text { activation of the PI3K/AKT } \\
\text { signal pathways }\end{array}$ & Intervene signal pathways & [4] \\
\hline $\begin{array}{l}\text { gene of phosphate and tension } \\
\text { homology deleted on } \\
\text { chromosome ten (PTEN) } \\
\text { mutations }\end{array}$ & $\begin{array}{l}\text { Gene deletion (gene } \\
\text { mutation) }\end{array}$ & [5] \\
\hline Axl overexpression & Abnormal gene expression & [5] \\
\hline KRAS, BRAF, HER-2 mutations & Gene mutation & [6] \\
\hline $\begin{array}{l}\text { MET factor proto oncogene } \\
\text { amplification and overexpression } \\
\text { of protein }\end{array}$ & Abnormal gene expression & [7] \\
\hline EMT & Deletion of signal pathways & [8] \\
\hline $\begin{array}{l}\text { membrane transporter-mediated } \\
\text { drug efflux pump mechanism, } \\
\text { such as P-gp, MRP, LRP }\end{array}$ & $\begin{array}{l}\text { Overexpressed membrane } \\
\text { protein with efflux pump, } \\
\text { related to pharmacokinetics }\end{array}$ & [9] \\
\hline $\begin{array}{l}\text { Intracellular abnormal enzyme } \\
\text { system }\end{array}$ & $\begin{array}{l}\text { Reduce drug activity and } \\
\text { enhances the DNA repair } \\
\text { ability }\end{array}$ & [10] \\
\hline $\begin{array}{l}\text { genes bcl-2 and c-myc } \\
\text { over-expression }\end{array}$ & Cell apoptosis & [11] \\
\hline
\end{tabular}

MiRNAs have been involved in a variety of signaling pathways, such as JNK/ELK1 signal pathway, PI3K/Akt signal pathway and NF-KB signaling pathway and so on. The capacity of a single miRNA to regulate the expression of multiple genes simultaneously presents an opportunity to use these small molecules in personalized therapy as individualized therapeutic tools. MiRNAs may not only be used as markers of the response to cisplatin but also as potential treatment tools that stimulate the sensitivity of lung cancer cells to this chemotherapeutic agent ${ }^{[84]}$. And they also have a very important guiding significance in avoiding and reversing lung cancer resistance effectively in the future. It is hoped that the miRNAs based targeting therapy can be applied in the future with more and more in-depth study of lung cancer cells and their drug resistance mechanism. Then we can effectively slow down the drug resistance of lung cancer patients and bring new opportunities for the treatment of patients, to improve a disappointing overall survival in lung cancer.

\section{Competing Interests}

The authors have declared that no competing interest exists.

\section{References}

[1] Lachgar A, Tazi MA, Afif M, et al. Lung cancer: Incidence and survival in Rabat, Morocco. Revue d'Épidémiologie et de Santé Publique. 2016;64:391-395.

[2] Osmani L, Askin F, Gabrielson E, et al. Current WHO guidelines and the critical role of immunohistochemical markers in the subclassification of non-small cell lung carcinoma (NSCLC): Moving from targeted therapy to immunotherapy. SEMIN CANCER BIOL. 2018;52:103-109.

[3] Siegel RL, Miller KD, Jemal A. Cancer Statistics, 2017. CA Cancer J Clin. 2017;67:7-30.
[4] Wang M, Sun ZY, Huang L. Research Progress on Secondary Resistance Mechanism of MiRNAs and EGFR-TKIs. Chinese Lung Cancer Journal. 2015;18:758-763.

[5] Lim WT, Zhang WH, Miller CR, et al. PTEN and phosphorylated AKT expression and prognosis in early- and late-stage non-small cell lung cancer. ONCOL REP. 2007;17:853.

[6] Pao W, Wang TY, Riely GJ, et al. KRAS mutations and primary resistance of lung adenocarcinomas to gefitinib or erlotinib. PLOS MED. 2005;2:e17.

[7] Engelman JA, Zejnullahu K, Mitsudomi T, et al. MET amplification leads to gefitinib resistance in lung cancer by activating ERBB3 signaling. SCIENCE. 2007;316:1039-1043.

[8] $\mathrm{Wu} \mathrm{F,} \mathrm{Li} \mathrm{J,} \mathrm{Jang} \mathrm{C,} \mathrm{et} \mathrm{al.} \mathrm{The} \mathrm{role} \mathrm{of} \mathrm{Axl} \mathrm{in} \mathrm{drug} \mathrm{resistance} \mathrm{and}$ epithelial-to-mesenchymal transition of non-small cell lung carcinoma. Int J Clin Exp Pathol. 2014;7:6653-6661.

[9] Wei H, Lu W, Li M, et al. Concomitance of P-gp/LRP Expression with EGFR Mutations in Exons 19 and 21 in Non-Small Cell Lung Cancers. YONSEI MED J. 2016;57:50-57.

[10] Huan Huang, Jihong Liu, Qinglian Meng, et al. Multidrug resistance protein and topoisomerase 2 alpha expression in non-small cell lung cancer are related with brain metastasis postoperatively. Int J Clin Exp Pathol. 2015;8:11537-11542.

[11] Javid J, Mir R, Mirza M, et al. CC genotype of anti-apoptotic gene BCL-2 $(-938 \mathrm{C} / \mathrm{A})$ is an independent prognostic marker of unfavorable clinical outcome in patients with non-small-cell lung cancer. CLIN TRANSL ONCOL. 2015;4:289-295.

[12] Morgillo F, Della Corte CM, Fasano M, et al. Mechanisms of resistance to EGFR-targeted drugs: Lung cancer. ESMO Open. 2016;1:e60.

[13] Shi Y, Au JS, Thongprasert S, et al. A prospective, molecular epidemiology study of EGFR mutations in Asian patients with advanced non-small-cell lung cancer of adenocarcinoma histology (PIONEER). J THORAC ONCOL. 2014;9:154-162.

[14] Zhong $\mathrm{W}$, Zhou $\mathrm{Q}, \mathrm{Wu} \mathrm{Y}$. The resistance mechanisms and treatment strategies for EGFR-mutant advanced non-small-cell lung cancer. Oncotarget. 2017;8:71358-71370.

[15] Ma Y, Pan X, Xu P, et al. Plasma microRNA alterations between EGFR-activating mutational NSCLC patients with and without primary resistance to TKI. Oncotarget. 2017;8:88529-88536.

[16] Wang J, Wang B, Chu $\mathrm{H}$, et al. Intrinsic resistance to EGFR tyrosine kinase inhibitors in advanced non-small-cell lung cancer with activating EGFR mutations. ONCOTARGETS THER. 2016;9:3711-3726.

[17] Zhao Y, Srivastava D. A developmental view of microRNA function. TRENDS BIOCHEM SCI. 2007;32:189-197.

[18] Ren P, Gong F, Zhang Y, et al. MicroRNA-92a promotes growth, metastasis, and chemoresistance in non-small cell lung cancer cells by targeting PTEN. Tumor Biology. 2016;37:3215-3225.

[19] George A. Calin, Carlo M. Croce. MicroRNA signatures in human cancers. Nat Rev Cancer. 2006;6:57-66.

[20] Thomas K. Sin, Fengfeng Wang, Fei Meng. Implications of microRNAs in the treatment of gefitinib-resistant non-small cell lung cancer. Int J Mol Sci. 2016;17:237.

[21] Namba K, Shien K, Takahashi Y, et al. Activation of AXL as a preclinical acquired resistance mechanism against osimertinib treatment in EGFR-Mutant non-small cell lung cancer cells. MOL CANCER RES. 2018.

[22] Chen L, Kong G, Zhang C, et al. MicroRNA-432 functions as a tumor suppressor gene through targeting E2F3 and AXL in lung adenocarcinoma. Oncotarget. 2016;7:20041.

[23] Wang Y, Xia H, Zhuang Z, et al. Axl-altered microRNAs regulate tumorigenicity and gefitinib resistance in lung cancer. CELL DEATH DIS. 2014;5:e1227.

[24] Cho C, Huang J, Shiah S, et al. Negative feedback regulation of AXL by miR-34a modulates apoptosis in lung cancer cells. RNA. 2016;22:303-315.

[25] Ghiso E, Migliore C, Ciciriello V, et al. YAP-Dependent AXL overexpression mediates resistance to EGFR inhibitors in NSCLC. NEOPLASIA. 2017;19:1012-1021.

[26] Zhang Z, Lee JC, Lin L, et al. Activation of the AXL kinase causes resistance to EGFR-targeted therapy in lung cancer. NAT GENET. 2012;44:852-860.

[27] Chunzhi Zhang, Lei Han, Anling Zhang, et al. MicroRNA-221 and microRNA-222 regulate gastric carcinoma cell proliferationand radioresistance by targeting PTEN. BMC CANCER. 2010;10:367.

[28] Xu HJ, Xu K, Zhou Y, et al. Enhanced tumor cell growth suppression by an n-terminal truncated retinoblastoma protein. Proc Natl Acad Sci USA. 1994:91:9837-9841.

[29] Tang JM, He QY, Guo RX, et al. Phosphorylated Akt overexpression and loss of PTEN expression in non-small cell lung cancer confers poor prognosis. LUNG CANCER. 2006;51:181-191.

[30] Yamamoto C, Basaki Y, Kawahara A, et al. Loss of PTEN expression by blocking nuclear translocation of EGR1 in gefitinib-resistant lung cancer 
cells harboring epidermal growth factor receptor-activating mutations. CANCER RES. 2010;70:8715-8725.

[31] Assia Derfoul, Aster H. Juan, Michael J. Difilippantonio, et al. De-creased microRNA-214 levels in breast cancer cells coincides with increased cell proliferation, invasion and accumulation of the Polycomb Ezh2 methyltransferase. CARCINOGENESIS. 2011;32:1607-1614.

[32] Xie X, Liu H, Mei J, et al. MiR-106a promotes growth and metastasis of non-small cell lung cancer by targeting PTEN. INT J CLIN EXP PATHO. 2015;8:3827.

[33] Stjernstrom A, Karlsson C, Fernandez OJ, et al. Alterations of INPP4B, PIK3CA and pAkt of the PI3K pathway are associated with squamous cell carcinoma of the lung. Cancer Med. 2014;3:337-348.

[34] Mariño G, Niso-Santano M, Baehrecke EH, et al. Self-consumption: The interplay of autophagy and apoptosis. NAT REV MOL CELL BIO. 2014;15:81-94.

[35] Scatena R, Bottoni P, Botta G, et al. The role of mitochondria in pharmacotoxicology: A reevaluation of an old, newly emerging topic. Am J Physiol Cell Physiol. 2007:293:C12-C21.

[36] Hacker G, Paschen SA. Therapeutic targets in the mitochondrial apoptotic pathway. Expert Opin Ther Targets. 2007;11:515-526.

[37] Christina Batsi, Soultana Mark opoulou, Evangelos Kontargiris, et al. Bcl-2 blocks 2-methoxyestradiol induced leukemia cell apoptosis by a p27Kip1-dependent $G_{1} /$ Scell cycle arrest in conjunction with NF- $\kappa$ B activation. BIOCHEM PHARMACOL. 2009;78:33-44.

[38] Armando Luna-López, Viridiana Y, González-Puertos, et al. A noncanonical NF- $\kappa$ B pathway through the p50 subunit regulates Bcl-2 overexpression during an oxidative-conditioning hormesis response. Free Radic Biol Med. 2013;63:41-50.

[39] Lin Zhang, Lihua Ming, Jian Yu. BH3 mimetics to improve cancer therapy:mechanisms and examples. Drug Resist Updat. 2007;10:207-217.

[40] Chittenden T, Harrington EA, O'Connor R, et al. Induction of apoptosis by the Bcl-2 homologue Bak. NATURE. 1995;374:733-736.

[41] Lalier L, Cartron P, Juin $\mathrm{P}$, et al. Bax activation and mitochondrial insertion during apoptosis. APOPTOSIS. 2007;12:887-896.

[42] Schwarz M, Andrade-Navarro MA, Gross A. Mitochondrial carriers and pores: Key regulators of the mitochondrial apoptotic program? APOPTOSIS. 2007;12:869-876.

[43] Shroff EH, Chandel N, Snyder C. Role of bcl-2 family members in anoxia induced cell death. CELL CYCLE. 2007;6:807-809.

[44] Elkholi R, Floros KV, Chipuk JE. The role of BH3-Only proteins in tumor cell development, signaling, and treatment. Genes \& Cancer. 2011;2:523-537.

[45] Liu G, Pei F, Yang F, et al. Role of autophagy and apoptosis in Non-Small-Cell lung cancer. INT J MOL SCI. 2017;18:367.

[46] Bergamaschi D, Samuels Y, O'Neil NJ, et al. IASPP oncoprotein is a key inhibitor of p53 conserved from worm to human. NAT GENET. 2003;33:162-167.

[47] Cryns V, Yuan J. Proteases to die for. Genes Dev. 1998;12:1551-1570.

[48] Cui R, Kim T, Fassan M, et al. MicroRNA-224 is implicated in lung cancer pathogenesis through targeting caspase-3 and caspase-7. Oncotarget. 2015;6:21802-21815.

[49] Wu D, Chen B, Cui F, et al. Hypoxia-induced microRNA-301b regulates apoptosis by targeting Bim in lung cancer. CELL PROLIFERAT. 2016:49:476-483.

[50] Fang JY, Richardson BC. The MAPK signalling pathways and colorectal cancer. LANCET ONCOL. 2005;6:322-327.

[51] Romano G, Acunzo M, Garofalo M, et al. MiR-494 is regulated by ERK1/2 and modulates TRAIL-induced apoptosis in non-small-cell lung cancer through BIM down-regulation. Proceedings of the National Academy of Sciences. 2012;109:16570-16575.

[52] Choi JY, Shin HJ, Bae IH. MiR-93-5p suppresses cellular senescence by directly targeting Bcl-w and p21. BIOCHEM BIOPH RES CO. 2018;505:1134-1140

[53] Kim EM, Jung CH, Kim J, et al. The p53/p21 complex regulates cancer cell invasion and apoptosis by targeting bcl-2 family proteins. CANCER RES. 2017;77:3092-3100.

[54] Baumgartner U, Berger F, Hashemi Gheinani A, et al. MiR-19b enhances proliferation and apoptosis resistance via the EGFR signaling pathway by targeting PP2A and BIM in non-small cell lung cancer. MOL CANCER. 2018;17:44

[55] Roulston A, Muller WJ, Shore GC. BIM, PUMA, and the achilles' heel of oncogene addiction. SCI SIGNAL. 2013;6:e12.

[56] Liu J, Chen L, Deng H, et al. Epithelial-to-mesenchymal transition in human esophageal cancer associates with tumor progression and patient's survival. INT J CLIN EXP PATHO. 2014;7:6943.

[57] Hannah, Farmer, Nuala, McCabe, Christopher J, Lord, et al. Targeting the DNA repair defect in BRCA mutant cells as a therapeutic strategy. NATURE. 2005:917-921.
[58] Bates RC, Bellovin DI, Brown C, et al. Transcriptional activation of integrin beta6 during the epithelial-mesenchymal transition defines a novel prognostic indicator of aggressive colon carcinoma. J CLIN INVEST. 2005;115:339-347.

[59] Burk U, Schubert J, Wellner U, et al. A reciprocal repression between ZEB1 and members of the miR-200 family promotes EMT and invasion in cancer cells. EMBO REP. 2008;9:582-589.

[60] Ahmad A, Maitah MY, Ginnebaugh KR, et al. Inhibition of Hedgehog signaling sensitizes NSCLC cells to standard therapies through modulation of EMT-regulating miRNAs. J HEMATOL ONCOL. 2013;6:77.

[61] Kong FF, Qu ZQ, Yuan HH, et al. Overexpression of FOXM1 is associated with EMT and is a predictor of poor prognosis in non-small cell lung cancer. ONCOL REP. 2014;31:2660-2668.

[62] Nichterlein T, Kretschmar M, Hof H. Fecal shedding of Listeria monocytogenes during murine listeriosis after intravenous infection. Zentralbl Bakteriol. 1994;281:192-195.

[63] Jonathon D. Roybal, Yi Zang, Young-Ho Ahn, et al. MiR-200 inhibits lung adenocarcinoma cell invasion and metastasis by targeting Flt1/VEGFR1. MOL CANCER RES. 2011;9:25-35.

[64] Adam L, Zhong M, Choi W, et al. MiR-200 expression regulates epithelial-to-mesenchymal transition in bladder cancer cells and reverses resistance to epidermal growth factor receptor therapy. CLIN CANCER RES. 2009;15:5060-5072.

[65] Xiao Liu, Yanhua Jia, Ke Zhang, et al. Construction of NCI-H3122 grams of azoltini resistant cell lines and preliminary screening of microRNAs associated with drug resistance in human non-small cell lung cancer. Journal of the Third Military Medical University. 2016;38:687-692.

[66] Chen H, Cheng X. Tumor heterogeneity and resistance to EGFR-targeted therapy in advanced nonsmall cell lung cancer: Challenges and perspectives. ONCOTARGETS THER. 2014;7:1689-1704

[67] Yoshida T, Song L, Bai Y, et al. ZEB1 mediates acquired resistance to the epidermal growth factor Receptor-Tyrosine kinase inhibitors in Non-Small cell lung cancer. PLOS ONE. 2016;11:e147344.

[68] Gao HX, Yan L, Li C, et al. MiR-200c regulates crizotinib-resistant ALK-positive lung cancer cells by reversing epithelial-mesenchymal transition via targeting ZEB1. MOL MED REP. 2016;14:4135-4143.

[69] Zhao YF, Han ML, Xiong YJ, et al. A miRNA-200c/cathepsin L feedback loop determines paclitaxel resistance in human lung cancer A549 cells in vitro through regulating epithelial-mesenchymal transition. ACTA PHARMACOL SIN. 2018;39:1034-1047.

[70] Zhen Q, Liu J, Gao L, et al. MicroRNA-200a targets EGFR and c-Met to inhibit migration, invasion, and gefitinib resistance in Non-Small cell lung cancer. CYTOGENET GENOME RES. 2015;146:1-8

[71] Feng X, Wang Z, Fillmore R, et al. MiR-200, a new star miRNA in human cancer. CANCER LETT. 2014;344:166-173.

[72] Shubin Wang, Guangjie Sui. Advances in the study of drug resistance in lung cancer mediated by membrane transporter protein. Journal of Applied Oncology. 2002;16:80-82.

[73] Kickhoefer VA, Rajavel KS, Scheffer GL, et al. Vaults are up-regulated in multidrug-resistant cancer cell lines. J BIOL CHEM. 1998;273:8971-8974.

[74] Scheffer GL, Wijngaard PL, Flens MJ, et al. The drug resistance-related protein LRP is the human major vault protein. NAT MED. 1995;1:578-582

[75] Kedersha NL, Rome LH. Isolation and characterization of a novel ribonucleoprotein particle: Large structures contain a single species of small RNA. J CELL BIOL. 1986;103:699-709.

[76] Janikova M, Zizkova V, Skarda J, et al. Prognostic significance of miR-23b in combination with P-gp, MRP and LRP/MVP expression in non-small cell lung cancer. NEOPLASMA. 2016;63:576-587.

[77] Fojo AT, Ueda K, Slamon DJ, et al. Expression of a multidrug-resistance gene in human tumors and tissues. Proc Natl Acad Sci U S A. 1987;84:265-269.

[78] Flens MJ, Zaman GJ, van der Valk P, et al. Tissue distribution of the multidrug resistance protein. AM J PATHOL. 1996;148:1237-1247.

[79] Izquierdo MA, Scheffer GL, Flens MJ, et al. Broad distribution of the multidrug resistance-related vault lung resistance protein in normal human tissues and tumors. AM J PATHOL. 1996;148:877-887.

[80] Schneikert J, Behrens J. The canonical Wnt signalling pathway and its APC partner in colon cancer development. GUT. 2007;56:417-425.

[81] Feng H, Zhang Z, Qing X, et al. Promoter methylation of APC and RAR-beta genes as prognostic markers in non-small cell lung cancer (NSCLC). EXP MOL PATHOL. 2016;100:109-113.

[82] Stewart DJ. Wnt signaling pathway in non-small cell lung cancer. J Natl Cancer Inst. 2014;106:t356.

[83] Holleman A, Chung I, Olsen RR, et al. MiR-135a contributes to paclitaxel resistance in tumor cells both in vitro and in vivo. ONCOGENE. 2011;30:4386-4398. 
[84] Florczuk M, Szpechcinski A, Chorostowska-Wynimko J. MiRNAs as biomarkers and therapeutic targets in Non-Small cell lung cancer: Current perspectives. TARGET ONCOL. 2017;12:179-200.

[85] Garofalo M, Quintavalle C, Di Leva G, et al. MicroRNA signatures of TRAIL resistance in human non-small cell lung cancer. ONCOGENE. 2008;27:3845-3855.

[86] Garofalo M, Jeon YJ, Nuovo GJ, et al. MiR-34a/c-dependent PDGFR- $\alpha$ / $\beta$ downregulation inhibits tumorigenesis and enhances TRAIL-induced apoptosis in lung cancer. PLOS ONE. 2015;10:e131729.

[87] Young-Jun Jeon, Justin Middleton, Taewan Kim, et al. A set of NF- $\kappa$ Bregulated microRNAs induces acquired TRAIL resistance in Lung cancer. Proc Natl Acad Sci U S A. 2015;112:E3355-E3364.

[88] Baretić D, Pollard HK, Fisher DI, et al. Structures of closed and open conformations of dimeric human ATM. SCI ADV. 2017;3:e1700933.

[89] Allison Stewart C, Tong P, Cardnell RJ, et al. Dynamic variations in epithelial-to-mesenchymal transition (EMT), ATM, and SLFN11 govern response to PARP inhibitors and cisplatin in small cell lung cancer. Oncotarget. 2017;8:28575.

[90] Lu J, Getz G, Miska EA, et al. MicroRNA expression profiles classify human cancers. NATURE. 2005;435:834-838.

[91] Lee Y, Jeon K, Lee JT, et al. MicroRNA maturation: Stepwise processing and subcellular localization. EMBO J. 2002;21:4663-4670. 